\title{
PRESERVACIÓN DE LOS
}

PETROGLIFOS QUILLACINGAS MEDIANTE LA INTEGRACIÓN DEL MOBILIARIO URBANO Y DE LA SIMBOLOGÍA INDÍGENA DESDE EL DISEÑO INDUSTRIAL

PRESERVATION OF THE QUILLACINGAS PETROGLYPHS THROUGH THE INTEGRATION OF URBAN FURNITURE AND INDIGENOUS SYMBOLS FROM INDUSTRIAL DESIGN

\section{Cesar Cadena Cortes}

Grupo de investigación

CORD, Facultad de Artes,

Universidad de Nariño.

williamcoral1996@gmail.

com

William Coral Chamorro

Grupo de investigación

CORD, Facultad de Artes,

Universidad de Nariño.

13 hotmail.com 


\section{Resumen}

En la investigación realizada en el PAT-LAB (Laboratorio de Patrimonio Cultural) a través de la estrategia desarrollada en la asignatura Seminario Trabajo de Grado, se identificó que en el entorno urbano de la ciudad de Pasto no existe ningún elemento arquitectónico, estatuas o monumentos que evoquen o transmitan información sobre las culturas milenarias que habitaron la región. La mayoría de estos se han estandarizado y no manejan una identidad local. Por tal razón, el PAT-LAB decidió indagar sobre la pertinencia de los petroglifos, ya que siendo patrimonio cultural inmueble (PCMU) son reconocidos como parte de la memoria y la identidad de las comunidades y grupos sociales históricos de la región. Debido a que la interpretación de los petroglifos está ligada a su ubicación, no es posible resguardarlos en sitios como museos y exposiciones. La intervención impulsada por la presente investigación, se tuvo que llevar a cabo en el entorno donde yacen los petroglifos. El objetivo de dicha intervención fue causar un impacto positivo en las personas y verlo reflejado en una mejora en la relación con el patrimonio. No obstante, con relación a los resultados, las estrategias que se han realizado para tratar esta situación no reflejan un cambio positivo y en la mayoría de los casos no se ha considerado intervenir, por lo cual en el presente proyecto se plantea fomentar el conocimiento sobre los petroglifos mediante una interacción con la comunidad desde el Diseño Industrial.

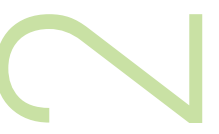

\section{Abstract}

In the research carried out at the PAT-LAB (Cultural Heritage Laboratory) through the strategy developed in the Undergraduate Work Seminar subject, it was identified that in the urban environment of the city of

Pasto there are no architectural elements, statues or monuments that evoke or transmit informatio about the ancient cultures that inhabited the region. Most of these have been standardized and do not handle a local identity. For this reason, the PAT$L A B$ decided to investigate the relevance of the petroglyphs, since being immovable cultural heritage (PCMU) they are recognized as part of the memory and identity of the communities and historical social groups of the region. Since the interpretation of the petroglyphs is linked to their location, it is not possible to protect them in places such as museums and exhibitions. The intervention promoted by this research had to be carried out in the environment where the petroglyphs lie. The objective of this intervention was to cause a positive impact on people and see it reflected in an improvement in the relationship with heritage. However, in relation to the results, the strategies that have been carried out to treat this situation do not reflect a positive change and in most cases it has not been considered to intervene, therefore in this project it is proposed to promote knowledge about petroglyphs through interaction with the community from Industrial Design. 

la sensación de inseguridad y el temor de visitar nuestro patrimonio y la

dificultad de acceso por parte de los organismos de protección, además de la destrucción del patrimonio en el fuego cruzado.

Intervenciones indebidas, aquí se encuentran registradas

- varias causas referentes al actuar humano, ya sea ocasionado intencionalmente o por falta de conocimiento.

Falta de apropiación social del patrimonio cultural, el

$\mathbf{A}$ lo largo de la historia de la humanidad, la forma constante de obtener conocimiento fue por medio del análisis de lo dejado por la generación anterior. físicos e inmateriales y de ahí viene el término de patrimonio, el cual hace alusión a la herencia cultural. Al desaparecer estos elementos, eventualmente el conocimiento también desaparecerá, una de las principales causas es la falta de sentido de pertenencia, lo cual causará una gran pérdida para todos porque al final no se sabría cuál fue el camino para llegar al lugar donde nos encontramos ahora y el legado de nuestras culturas indígenas se perdería poco a poco.

Según el ministerio de cultura en Colombia, las razones por las cuales el patrimonio inmueble puede llegar a deteriorarse o incluso perderse de manera irreversible, se pueden clasificar en 3 categorías principales, las cuales son: Eventos naturales, inducidos o accidentales, esta causa es dada por fenómenos naturales o por la intervención humana indebida, como la minería, industria, ocupación, entre otros. Esto genera que se dificulte el acceso hacia los monumentos, también se pueden producir cambios en la dinámica natural.

Conflicto social o armado actualmente en Colombia, existen grupos armados que se adueñan de territorios en los cuales, debido a esta problemática, la protección del patrimonio es la menor preocupación en la comunidad y el gobierno, en la mayoría de estas zonas en las que se observa este fenómeno se pierden muchos referentes de identidad. algunas estrategias de mitigación, consiste en fomentar alternativas de emprendimiento a partir del patrimonio, el fortalecimiento de la protección a nivel nacional y la aplicación de desconocimiento del patrimonio en general y la falta de formación básica en este tema por parte de las instituciones educativas, ocasiona la pérdida de la identidad en las regiones junto a su comunidad, la descontextualización y desarticulación del patrimonio cultural como fuente de desarrollo y por consiguiente algunas personas llegan a percibir al patrimonio como un obstáculo hacia el desarrollo social-económico. también es evidente un deterioro material y de valores, ya que no se realiza un mantenimiento según normativas y falta realizar acciones preventivas para la conservación de los bienes de interés cultural.

Insuficiencia técnica y especializada en el área de salvaguardar los patrimonios, la cantidad de profesionales idóneos en este campo no es suficiente para cubrir todas las necesidades del territorio, además de que con el paso del tiempo se han ido perdiendo las técnicas de construcción tradicionales necesarias para un buen mantenimiento del patrimonio. no se están aplicando los principios de conservación y protección del patrimonio cultural, porque en muchos casos se realiza una intervención empírica, lo que ocasiona un detrimento irreparable.

Desconocimiento de la normativa, son varias las problemáticas en cuanto a la normativa, debido a que los entes territoriales no aplican un control eficiente y la divulgación de dicha normativa es insuficiente, lo que provoca una inoperancia administrativa, inestabilidad jurídica, confusión respecto a la normativa aplicable, presencia tardía de las autoridades competentes, actuaciones contradictorias y la ejecución de intervenciones al margen de la ley.

(MINCULTURA) Faltas contra el patrimonio.

De acuerdo con lo anterior, proteger el patrimonio cultural e histórico de un territorio es proteger el conocimiento que este resguarda. Tal es el objetivo de las organizaciones mundiales como 
la Unesco cuando otorgan títulos de patrimonio, es justamente el de proteger el conocimiento. Entre los elementos protegidos de la humanidad se encuentran los petroglifos, los cuales además son de carácter inmueble, es decir, que su ubicación hace parte del significado global (Unesco, 2004; ICANH, 2010). Sin embargo, no basta solo con nombrar como patrimonio a estos elementos culturales, ya que se requiere de todo un sistema logístico para que la sociedad entienda el valor de un patrimonio y se logre asimilar como algo propio. En efecto, cuando la comunidad que habita o frecuenta dicho territorio no posee un sentido de pertenencia, el patrimonio tiende a ser destruido por las mismas personas, como ya ha ocurrido con algunos petroglifos (Maldonado, 2018; Calderón et al, 2018). La solución no es simplemente resguardar a los petroglifos de la sociedad porque, aunque se preservaron durante mucho tiempo, las personas no tienen un conocimiento de la importancia de estos elementos y lo que se busca con el patrimonio en sí es seguir transmitiendo conocimiento a través del paso del tiempo.

Sea como fuere, la mayoría de estrategias de los referentes en otros lugares del mundo, consiste en plasmar representaciones de su patrimonio en zonas bastante concurridas como parques, templos, terminales terrestres y aéreas, entre otros. De esta manera, los habitantes logran fomentar la cultura local y generar ingresos monetarios. Actualmente en la ciudad de Pasto no se desarrollan ni existen proyectos para fomentar la conexión social con los patrimonios de forma activa, sector fundamental que se está desaprovechando. A causa de que no es recomendable hacer turismo en torno a los petroglifos actualmente, ya que aún no existen las herramientas o el sentido de cuidado de estos, el equipo de investigación planteó otras alternativas a través del análisis del presente proyecto. Por ejemplo, varios empleos nuevos se podrían generar a partir del mantenimiento y cuidado de zonas con petroglifos.

Al respecto, el geógrafo Carlos Erazo dice que es importante saber el significado de los petroglifos y generar una resignificación de la noción de comunidad para así crear nuevamente dinámicas con estos elementos (Conversación con C. Erazo. 17 de marzo del 2020). Al acercar estos conocimientos y promover el sentido de pertenencia a la ciudadanía en general, se fomentará la visita de forma responsable a los petroglifos, lo que le dará mayor visibilidad hacia la sociedad y los entes gubernamentales, que podrían desarrollar propuestas de preservación directas en estos sitios junto a las comunidades indígenas que se ubican allí, lo que fomentaría una mejora en su calidad de vida. Asimismo, es importante abordar el tema de los petroglifos desde la disciplina del Diseño Industrial, ya que esto fomenta el estudio desde este campo y genera muchas otras formas de abordar las problemáticas que pueden ser tratadas desde las disciplinas creativas. En otras palabras, mediante el Diseño Industrial se pueden generar estrategias que ayuden a fomentar y crear sentido de pertenencia respecto a nuestro patrimonio cultural para que, de manera simultánea, se contribuya a su preservación.

\section{Metodología}

\section{Características de la investigación-creación}

Según Ballesteros y Beltrán (2018) Nos damos cuenta que en los procesos creativos, especialmente de investigación-creación, es muy común avanzar y regresar en cada etapa del proyecto. Esto es lo que se conoce como iteración. La iteración permite al investigador en diseño experimentar y avanzar en el proceso creativo, ajustar cada elemento al analizar los resultados obtenidos, mediante la exploración rigurosa se analiza y evalúa los resultados obtenidos, lo que conduce a elegir las mejores propuestas. Por último, el lenguaje plástico y sensorial son las formas creativas en las que se da a conocer los resultados obtenidos con cierta libertad en las herramientas de transmisión de la información.

Contextualización es identificar y apropiar un conocimiento que permitirá hacer inteligible los aspectos que determinan el tema correspondiente. Factor que contribuye a identificar los procesos que se han desarrollado al respecto y a abordar el problema desde diferentes perspectivas. Después de dicha fase, la sensación detonante es el momento en el que, después de analizar e interpretar la información obtenida en la contextualización, se comienza a identificar los elementos clave que lo rodean y también los posibles enfoques de trabajo de acuerdo al interés del diseñador. A partir de dicha fase se generan las posibles ideas con respecto a la problemática planteada. Para esta parte de la investigación-creación se generarán procesos de acercamiento a la comunidad indígena quillacinga, para crear un espacio de participación que enriquezca el proceso de diseño. 
Conformación Plástica es cuando llega el momento de materializar y generar la transmisión del mensaje, cuando todo el proceso que venimos realizando debe plasmarse en un elemento capaz de transmitir

toda la información, concepto y trabajo realizado.
Para la obtención y recolección de datos relevantes se llevaron a cabo una serie de entrevistas semi-estructuradas y visitas de campo con evidencia fotográfica. Las entrevistas se hicieron a especialistas del tema de estudio, para que la información obtenida se basará en estándares académicos y datos empíricos. Por último, vale la pena mencionar que una buena parte de la información surge como producto de la observación en el trabajo de campo y de la interpretación de los petroglifos y de la interacción de la gente con los mismos.

\section{Resultados}

Como resultado parcial se hizo un documento recopilatorio en el cual se identificaron los elementos y estrategias que puedan utilizarse como un posible sistema de apropiación. Asimismo, esto permitió evaluar la viabilidad de las estrategias para poder transmitir la simbología indígena y el cuidado de los petroglifos a la sociedad de la manera más idónea. Tal como lo plantean Castañeda y Cardona (2016), una vez hecho el análisis y la evaluación se hicieron diferentes propuestas objetuales orientadas a la apropiación cultural de la simbología indígena correspondiente a los petroglifos en el entorno urbano. Así, se hizo un análisis de los elementos de interacción urbanos que puedan generar una apropiación de conocimiento (Suárez \& Laguna, 2017). A continuación se muestran los modelados en 3D del sistema propuesto.

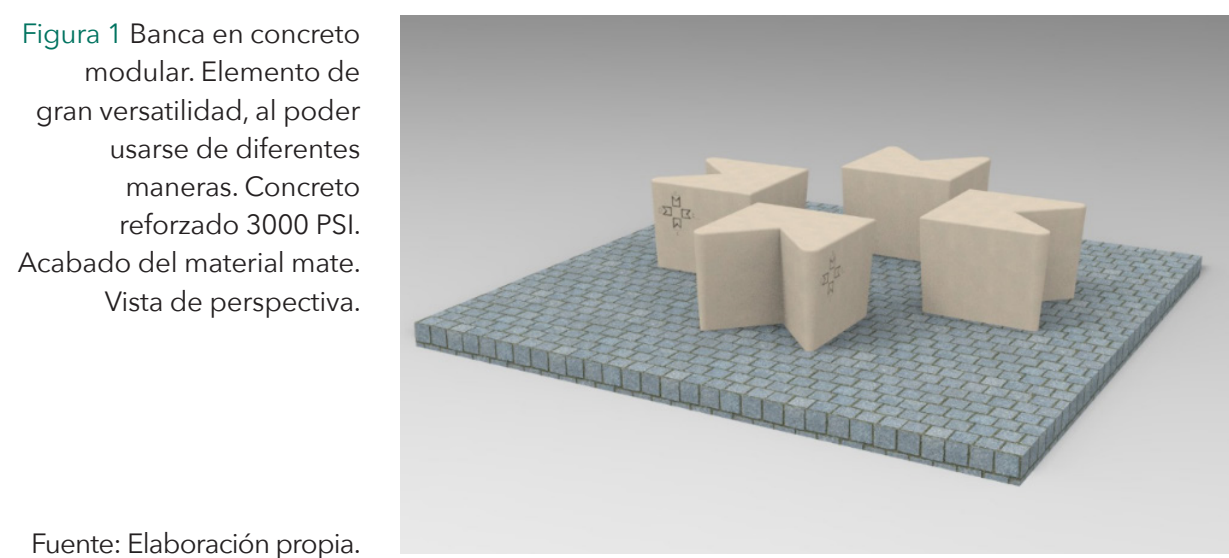

Figura 2 Poste de alumbrado público. Brazo y poste principal en tubo metálico, globos de policarbonato $+1$ $+$ (n) 


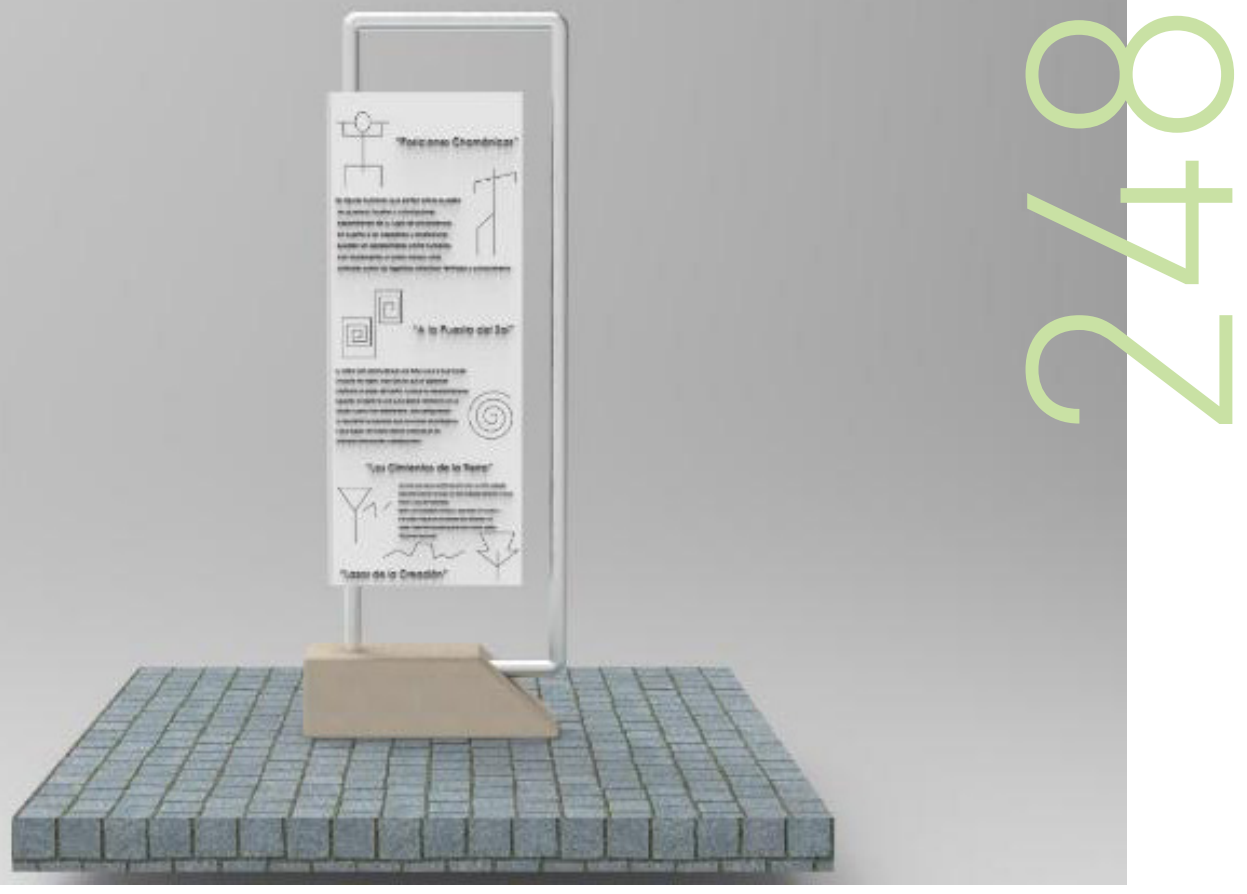

Figura 5 Tótem informativo Fuente: Elaboración propia. Tótem realizado en lámina de acero inoxidable. Estructura en tubería metálica de $2^{\prime \prime}$ de diámetro. Base en cemento 3000 PSI. Acabado módulo principal acabado del material con aplicación de pintura electrostática blanca RAL 9010. Vista de perspectiva.

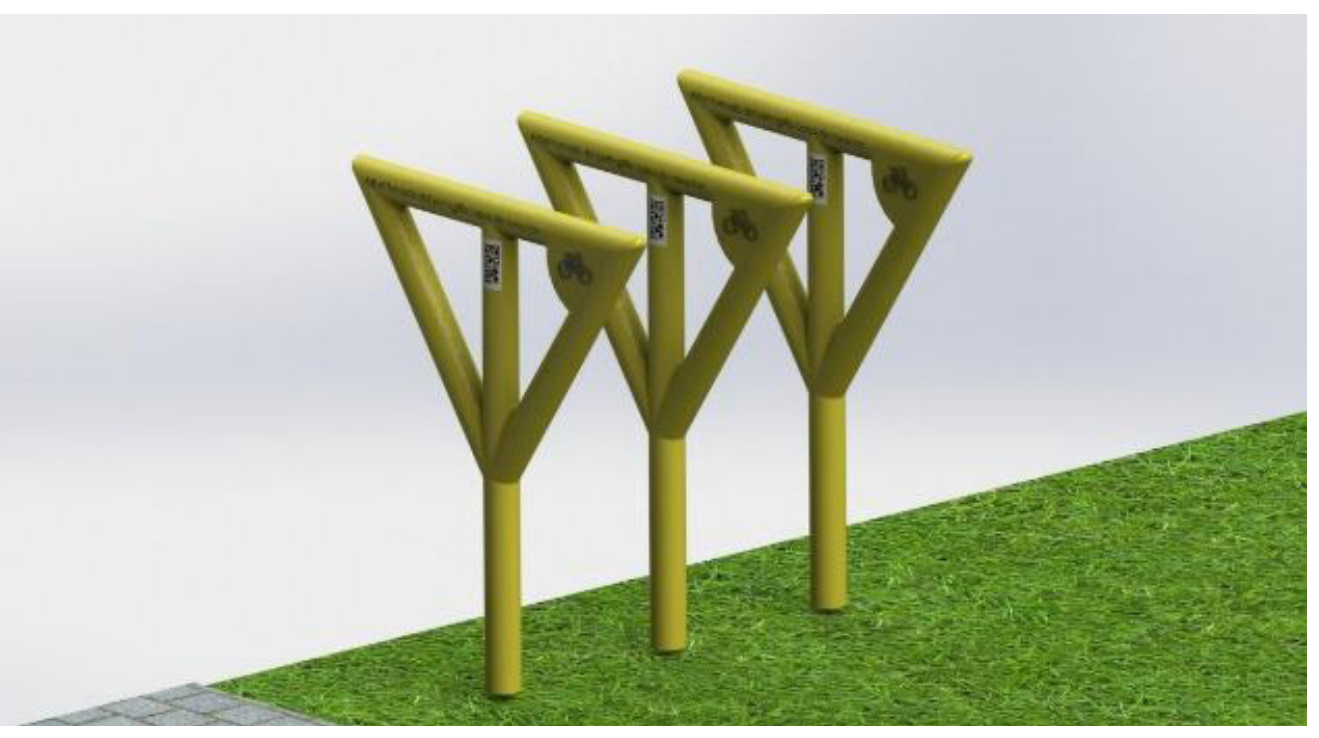

Figura 6 Paradero de bicicleta Fuente: Elaboración propia. Elemento de tubo metálico con tubería en ángulos de $45^{\circ}$ de $2^{\prime \prime}$ el diámetro. Pintura electrostática amarillo RAL

$$
\text { 1060. Vista de perspectiva. }
$$

Conclusiones

Al aun no implementarse el proyecto no se ha podido comprobar el desarrollo de sentido de pertenencia en la comunidad, pero la formulación de propuestas objetuales y su puesta en un espacio mediante los modelados nos permite seguir en la búsqueda de mejores alternativas de diseño lo que nos acerca cada vez más a cumplir los objetivos del proyecto y su mejor desarrollo.

Al generar un interés en la ciudadanía sobre los petroglifos, fomentará que el Estado y la comunidad local creen estrategias de cuidado y aprovechamiento sostenible de este patrimonio. Asimismo, la presente investigación puede abrir el interés de otros investigadores y estudiantes por indagar sobre temáticas relacionadas con el patrimonio cultural y proyectos de investigación-creación.

Por otro lado, el equipo de investigación piensa que la comunidad en general va a adquirir un sentido de pertenencia, lo que fomentará el respeto y el cuidado a los lugares donde se encuentran los petroglifos. Por medio de los artefactos desarrollados, se generará una interacción con los usuarios que fomentará el uso de los espacios públicos y también la visita responsable de algunos lugares donde se ubican los petroglifos como actividad lúdica y cultural, lo que podría ser beneficioso para las comunidades locales.

Las comunidades indígenas tienen un sentido de cuidado y pertenencia de estos, pues para ellos es un núcleo de culto e identidad cultural, cuando es posible algo que hacen estas comunidades es construir instituciones alrededor de los petroglifos. De esta forma, pueden visitar y realizar cultos sagrados y resguardarlos. Pero no es solo trabajo de estas comunidades preservar y cuidar estos elementos, sino también de toda la ciudadanía.

Referencias bibliográficas

Ballesteros. M. \& Beltrán. E. (2018). ¿Investigar creando? una guía para la investigación-creación en la academia. Bogotá D.C: Universidad del Bosque.

Cardona O. \& Castañeda G. (2016). Diseño como estrategia de visibilización del patrimonio cultural de una comunidad en contexto. XIII Foro académico de Diseño. Universidad de Caldas, Manizales. 
Ministerio de Cultura. (2015). Nuestro Patrimonio Cultural está al alcance de todos. Min Cultura https://www.mincultura.gov. co/areas/patrimonio/preguntas-frecuentes/Paginas/default. aspx.

Calderón, B. (2018). El reto de enseñar y comunicar el patrimonio en el Siglo XXI. En B. Calderón (Coord.), A. Choque Porras (Coord.), F. Quiles García (Coord.). Nuevas tecnologías e interdisciplinariedad en la comunicación del patrimonio cultural. Sevilla: Enredars.

ICANH. (2010). Lineamientos constitucionales y legales para la protección del patrimonio arqueológico colombiano. Recuperado de: https://www.icanh.gov.co/recursos_user// ICANH\%20PORTAL/OTROS\%20ARCHIVOS/Circular\%20 a\%20Entidades\%20territoriales\%2012-02-10\%20(2).pdf

Maldonado, R. (2018). El diseño industrial, salvaguardia del patrimonio cultural inmaterial en México. Economía Creativa, (10), 55-87.

Suárez S. \& Laguna L. (2017). Estudio descriptivo de los petroglifos presentes en el municipio de Arboleda, Nariño como referente morfológico para la aplicación de identidad corporativa (Tesis de pregrado). Universidad Autónoma de Occidente, Cali. UNESCO. (2004). La UNESCO y el Patrimonio Mundial. Recuperado de: http://www.unescoetxea.org/ dokumentuak/UNESCOPatrimonio.pdf 